tested if alterations in dysbindin mRNA were found in the hippocampus of patients with schizophrenia by using in situ hybridization. We found significantly reduced dysbindin mRNA in the dentate gyrus, CA4 and CA3, but not CA1, subregions of the hippocampus of patients with schizophrenia as compared with normal controls. Additionally, we found that dysbindin mRNA levels strongly and positively correlated with synaptophysin and spinophilin mRNA levels, which are known to be reduced in patients with schizophrenia. Our results suggest that reductions in dysbindin protein previously found in the hippocampus of patients with schizophrenia may be because of decreased dysbindin mRNA. The significant reduction of dysbindin mRNA found in the hippocampus confirms and extends our initial findings that dysbindin mRNA is significantly reduced in the frontal cortex and tends to be decreased in the midbrain of patients with schizophrenia (Weickert et al. 2004). Taken together, our results suggest that dysbindin mRNA reduction is not anatomically restricted, but may be anatomically specific, in the brains of patients with schizophrenia. Furthermore, subfield-specific reductions in dysbindin mRNA may lead to subfield-specific synaptic pathology in the hippocampus of patients with schizophrenic.

\section{The Stress Sampler Study II: psychological functioning and coping within a diabetes sample}

\section{T Showyin 1,2, K Baikie ${ }^{1,2}$, A Finch $^{1,2}$, P Mitchell', G Parker ${ }^{1,2}$, J Reddy ${ }^{1}$, PR Schofield ${ }^{3}$, L Wedgwood ${ }^{1,2}$, K Wilhelm ${ }^{1,4}$ \\ 'School of Psychiatry, University of New South Wales; ${ }^{2}$ The Black Dog Institute, Sydney, Australia; 'Prince of Wales Medical Research Institute; and \\ ${ }^{4}$ Consultation Liaison Psychiatry, St Vincent's Hospital, Sydney, Australia}

Background: Diabetes is a complex illness that involves ongoing monitoring and management. The stress of adjusting one's lifestyle to comply with the demands of the illness can often impact on an individual's mental health, therefore increasing the need for effective coping efforts. This paper aims to identify coping styles associated with positive psychological adjustment in a sample of hospital out-patients with diabetes mellitus.

Method: The Stress Sampler Study commenced in July 2006 and recruitment is ongoing at a hospitalbased diabetes clinic. The baseline measures include the Patient Health Questionnaire (which is based on DSM-IV diagnostic criteria), K-10 (psychological distress), SF-12 (physical and psychological self-report of functioning), clinical interview and the COPE (which measures a variety of coping responses).

Results and Conclusions: Preliminarily data will be presented on psychological distress and functioning and the association with current coping responses within this diabetic sample $(n>100)$. It is anticipated that these findings will contribute to our understanding of positive coping efforts associated with psychological adjustment in people with diabetes and may inform the education of diabetes management.

\section{[3H]Pirenzepine binding in the pathology of schizophrenia: studies in Brodmann's area 6}

\section{A Soulby, B Dean, E Scarr \\ Mental Health Research Institute, Melbourne, Australia}

Background: Changes in muscarinic receptors are widespread in the brains of subjects with schizophrenia, implicating them in the pathology of the disorder (Hyde \& Crook Chem Neuroanat 2001, 22 53-63). A previous study showed decreased levels of mRNA for the muscarinic M1 receptor in Brodmann's area (BA) 6 from subjects with schizophrenia (Mancama et al. Am J Med Genet B Neuropsychiatr Genet 2003, 119 $2-6)$. The purpose of our study was to further investigate this finding.

Methods: Using in situ radioligand binding and autoradiography, the density of [3H]pirenzepine binding was determined in BA 6 obtained postmortem from 20 subjects with schizophrenia and 20 subjects with no psychiatric history (controls).

Results: $[3 \mathrm{H}]$ Pirenzepine binding was differentially distributed in the cortical gray matter (inner layer = gray1; outer layer $=$ gray2). [3H]Pirenzepine binding was significantly lower in tissue from subjects with schizophrenia than in that from control subjects in both gray 1 (mean \pm SEM, $74.56 \pm 10.67$ vs. $118.80 \pm 5.47$ fmole/mgETE, $P<0.01)$ and gray2 layers $(102.90 \pm 14.09$ vs. $152.90 \pm 8.04$, $P<0.01)$. Furthermore, $[3 \mathrm{H}]$ pirenzepine binding was significantly different between layers in control subject tissue (gray1, $118.80 \pm 5.47$; gray2, $152.90 \pm 8.04 ; P<0.05)$, but not in that from subjects with schizophrenia (gray1, $74.56 \pm 10.67$; gray2 $102.90 \pm 14.09 ; P>0.05)$.

Conclusion: These results support previous findings that levels of M1 and/or M4 muscarinic receptors are significantly altered in cortical regions from subjects with schizophrenia, extending the regions affected to include BA6. 